\title{
Angiopoietin-1, Angiopoietin-2 and Myocardial Ischemia-Reperfusion Injury
}

\section{Chen Baoxia*}

Cardiology Department, Peking University Third Hospital, China

Prolonged periods of myocardial ischemia cause irreversible myocardium loss. Thus, early reperfusion is the paramount strategy for myocardial salvage. However, reperfusion itself also causes myocardial damage known as reperfusion injury [1]. Angiopoietin-1 (Ang-1) and angiopoietin-2 (Ang-2), initially implicated in embryonic vasculogenesis and angiogenesis [2,3], were shown to be involved in myocardial ischemia/reperfusion (I/R) injury in recent years $[4,5]$.

\section{Angiopoietins}

Angiopoietins have been identified in the mid 1990s as a family of growth factors that are essential for blood vessel formation. There are four types of angiopoietins: angiopoietin-1 (Ang-1), angiopoietin-2 (Ang-2), angiopoietin-3 (Ang-3), and angiopoietin-4 (Ang-4). Angiopoietins are secreted glycoproteins with a dimeric molecular weight of approximately $75 \mathrm{kDa}$. The best characterized angiopoietins are Ang-1 and Ang-2 [2,3]. Very little is known about Ang-3 and Ang4 [6]. Ang-1 is primarily expressed by mesenchymal cells. Ang-2 is almost exclusively expressed by endothelial cells where it is stored in Weibel-Palade bodies (WPB) [2,3]. Following endothelium activation, Ang-2 is rapidly released from WPB [7].

Angiopoietins are ligands binding to the tyrosine kinase receptor Tie-2, which is present on endothelial cells and neutrophils $[2,3,6]$. Ang- 1 acts in a stimulating, agonistic manner on Tie2, whereas Ang2 exerts antagonistic functions on Ang-1/Tie2 signaling [2,3]. Some studies also identified Ang-2 as an agonist of Tie2 [8]. It has been suggested that Ang-1 could also exert its activity in non-endothelial cells through integrins [5,9].

\section{Pathophysiologic Mechanisms of Myocardial I/R Injury and the Relevance of Angiopoietins}

Molecular and cellular events underlying I/R are complex. The inflammatory response partially mediates $\mathrm{I} / \mathrm{R}$ injury [1]. Endothelial activation and injury increase vascular permeability, leading to interstitial edema and subsequently compounding myocardium loss [1]. Cellular adhesion molecules elicited by the injured endothelium (e.g., intercellular adhesion molecule-1 (ICAM-1), vascular cell adhesion molecule-1 (VCAM-1), E-selectin) promote tissue invasion by inflammatory cells. These infiltrating cells, including (and in particular) neutrophils, are directly toxic to the myocardium by secreting proteases, generating reactive oxygen species (ROS), and occluding the microvasculature [1]. Ang-1 has been shown to inhibit vascular permeability and exert anti-inflammatory effect via the Tie-2 receptor. Ang-1 attenuates thrombin-induced permeability in human pulmonary microvascular endothelial cells (HPMVECs) by enforcing the VE-cadherin organization [10]. Ang-1 could counteract the inflammatory effects of vascular endothelial growth factor (VEGF) on endothelial cells [11], ameliorate ROS-induced acute lung injury [12] and lipopolysaccharide-induced acute kidney injury [13] by reducing the induction of ICAM-1 and VCAM-1, causing a reduction in leukocyte adhesion and subsequent inflammation. While Ang-1 has been shown to have a protective effect against inflammation, Ang2 has been shown to have the opposite effect. Ang- 2 could sensitize thrombin-induced permeability in HPMVECs by destabilizing VEcadherin junctions and increasing gap formation [10]. Overexpression of Ang-2 also sensitizes ICAM-1 and VCAM-1 expression in diabetic mouse hearts [14]. Osteoprotegrin (OPG) has been shown to up regulate Ang-2 in endothelial cells thereby sensitizing the cells to tumor necrosis factor- $\alpha$ (TNF- $\alpha$ ) [15]. TNF- $\alpha$ is a pro-inflammatory cytokine which stimulates adhesion molecule up regulation.

Apoptosis has been observed in hearts subjected to either continuous ischemia or ischemia followed by reperfusion [1] Ang-1 promotes cell survival. Ang-1 binds to Tie2 on endothelial cells, leading to phosphorylation of phosphatidylinositol 3-kinase (PI3 K). PI3 K activates Akt, and stimulates the phosphorylation and thereby the inhibition of pro-apoptotic proteins, including BAD and procaspase- 9 [16]. Additionally, Akt upregulates survivin, a classical apoptosis inhibitor, and thereby supports cell survival [17]. Ang-1 has also been shown to promote cardiac and skeletal myocyte survival through integrins $^{5}$. Unlike a general agreement about the influence of Ang-1 on cell survival, there are conflicting results regarding the physiological roles of Ang-2 [18,19]. Ang-2 may promote both endothelial cell survival and apoptosis depending on VEGF concentrations.

\section{Evidence from Animal and Human Studies}

In order to investigate the participation of Angiopoietins in myocardial $\mathrm{I} / \mathrm{R}$, a previous study using a rat model of left anterior descending coronary artery (LAD) occlusion and reperfusion, has shown that expression of Ang2 increased after I/R in the ventricular myocardium, while the expression of Ang1 did not [4]. We investigated serum Ang- 1 and Ang- 2 levels in 85 patients with ST-segment elevation myocardial infarction (STMEI), and found that Ang-1, Ang-2 and Ang-2/Ang-1 ratio (Ang-2/1) were all significantly elevated. This study also showed that Ang-2 and Ang-2/1 were positively correlated with peak cardiac troponin $\mathrm{T}$ levels, suggesting that the extent of myocardial damage may be linked to circulating Ang-2 and Ang-2/1[20]. In the study by Lee et al. [5], shifting of the Ang-2/1 ratio to favor Ang1 by administration of adenovirus expressing Ang- 1 in a mouse I/R model could prevent vascular leakage through regulating VE-cadherin phosphorylation, increase cardiomyocyte survival and reduce infarct size [5].

In conclusion, Ang-1 has anti-permeability, anti-inflammatory

*Corresponding author: Chen Baoxia, Associate Chief Physician Cardiology Department, Peking University Third Hospital, China, E-mail: chenbaoxia@medmail.com.cn

Received October 26, 2012; Accepted October 27, 2012; Published October 29 2012

Citation: Baoxia C (2012) Angiopoietin-1, Angiopoietin-2 and Myocardial IschemiaReperfusion Injury. J Hypertens 1:e107. doi:10.4172/2167-1095.1000e107

Copyright: (c) 2012 Baoxia C. This is an open-access article distributed under the terms of the Creative Commons Attribution License, which permits unrestricted use, distribution, and reproduction in any medium, provided the original author and source are credited. 
Citation: Baoxia C (2012) Angiopoietin-1, Angiopoietin-2 and Myocardial Ischemia-Reperfusion Injury. J Hypertens 1:e107. doi:10.4172/21671095.1000e107

and anti-apoptosis effects, and is protective in I/R injury. Further work is needed to better understand the pathological effect of Ang-2 on endothelial cell and cardiomyocyte survival. Human studies suggested that the extent of myocardial damage may be linked to circulating Ang2 and Ang-2/1 in AMI patients. Therefore, further studies are needed to clarify the role of Ang-2 in I/R injury.

\section{References}

1. Turer AT, Hill JA (2010) Pathogenesis of myocardial ischemia-reperfusion injury and rationale for therapy. Am J Cardiol 106: 360-368

2. Davis S, Aldrich TH, Jones PF, Acheson A, Compton DL, et al. (1996) Isolation of angiopoietin-1, a ligand for the TIE2 receptor, by secretion-trap expression cloning. Cell 87: 1161-1169.

3. Maisonpierre PC, Suri C, Jones PF, Bartunkova S, Wiegand SJ, et al. (1997) Angiopoietin-2, a natural antagonist for Tie2 that disrupts in vivo angiogenesis. Science 277: 55-60.

4. Shyu KG, Chang CC, Wang BW, Kuan $P$, Chang $H$ (2003) Increased expression of angiopoietin-2 and Tie2 receptor in a rat model of myocardial ischaemia/ reperfusion. Clin Sci (Lond) 105: 287-294.

5. Lee SW, Won JY, Lee HY, Lee HJ, Youn SW, et al. (2011) Angiopoietin-1 protects heart against ischemia/reperfusion injury through VE-cadherin dephosphorylation and myocardiac integrin-ß1/ERK/caspase-9 phosphorylation cascade. Mol Med 17: 1095-1106.

6. Valenzuela DM, Griffiths JA, Rojas J, Aldrich TH, Jones PF, et al. (1999) Angiopoietins 3 and 4: diverging gene counterparts in mice and humans. Proc Natl Acad Sci U S A 96: 1904-1909.

7. Fiedler U, Scharpfenecker M, Koidl S, Hegen A, Grunow V, et al. (2004) The Tie-2 ligand angiopoietin-2 is stored in and rapidly released upon stimulation from endothelial cell Weibel-Palade bodies. Blood 103: 4150-4156.

8. Veikkola T, Alitalo K (2002) Dual role of Ang2 in postnatal angiogenesis and lymphangiogenesis. Dev Cell 3: 302-304.

9. Dallabrida SM, Ismail NS, Pravda EA, Parodi EM, Dickie R, et al. (2008) Integrin binding angiopoietin-1 monomers reduce cardiac hypertrophy. FASEB J 22: 3010-3023.

10. van der Heijden M, van Nieuw Amerongen GP, van Bezu J, Paul MA,
Groeneveld AB, et al. (2011) Opposing effects of the angiopoietins on the thrombin-induced permeability of human pulmonary microvascular endothelia cells. PLoS One 6: e23448.

11. Kim I, Moon SO, Park SK, Chae SW, Koh GY (2001) Angiopoietin-1 reduces VEGF-stimulated leukocyte adhesion to endothelial cells by reducing ICAM-1, VCAM-1, and E-selectin expression. Circ Res 89: 477-479.

12. Kim SR, Lee KS, Park SJ, Min KH, Lee KY, et al. (2008) Angiopoietin-1 variant COMP-Ang1 attenuates hydrogen peroxide-induced acute lung injury. Exp Mo Med 40: 320-331.

13. Kim DH, Jung YJ, Lee AS, Lee S, Kang KP, et al. (2009) COMP-angiopoietin-1 decreases lipopolysaccharide-induced acute kidney injury. Kidney Int 76: 11801191.

14. Chen JX, Zeng H, Reese J, Aschner JL, Meyrick B (2012) Overexpression of angiopoietin-2 impairs myocardial angiogenesis and exacerbates cardiac fibrosis in the diabetic $\mathrm{db} / \mathrm{db}$ mouse model. Am J Physiol Heart Circ Physio 302: $\mathrm{H} 1003-\mathrm{H} 1012$.

15. Mangan SH, Van Campenhout A, Rush C, Golledge J (2007) Osteoprotegerin upregulates endothelial cell adhesion molecule response to tumor necrosis factor-alpha associated with induction of angiopoietin-2. Cardiovasc Res 76 494-505.

16. Kim I, Kim HG, So JN, Kim JH, Kwak HJ, et al. (2000) Angiopoietin-1 regulates endothelial cell survival through the phosphatidylinositol 3'-Kinase/Akt signal transduction pathway. Circ Res 86: 24-29.

17. Papapetropoulos A, Fulton D, Mahboubi K, Kalb RG, O'Connor DS, et al (2000) Angiopoietin-1 inhibits endothelial cell apoptosis via the Akt/survivin pathway. J Biol Chem 275: 9102-9105.

18. Kim I, Kim JH, Moon SO, Kwak HJ, Kim NG, et al. (2000) Angiopoietin-2 at high concentration can enhance endothelial cell survival through the phosphatidylinositol 3'-kinase/Akt signal transduction pathway. Oncogene 19 : 4549-4552.

19. Harfouche R, Hussain SN (2006) Signaling and regulation of endothelial cel survival by angiopoietin-2. Am J Physiol Heart Circ Physiol 291: H1635-H1645.

20. Chen S, Guo L, Cui M, Sun L, Mi L (2012) Dynamic changes in serum angiopoietin-1, angiopoietin-2, and angiopoietin-2/angiopoietin-1 ratio in acute myocardial infarction patients treated with primary percutaneous coronary intervention. Biomarkers 17: 441-446. 